\title{
Hypoglycemic Properties of Some Local Herbs Extracts in Streptozotocin-Induced Diabetic Wistar Albino Rats
}

\author{
${ }^{1}$ G.S. George, PhD; ${ }^{2}$ Prof. A.A. Uwakwe \\ ${ }^{I}$ Department of Medical Laboratory Science Niger Delta University, Wilberforce Island, Bayelsa State, Nigeria. \\ ${ }^{2}$ Department of BiochemistryUniversity of Port Harcourt, Nigeria.
}

\begin{abstract}
The effects of aqueous extract of Tapinanthus bengwensis and Ocimum gratissimum treatment on Wistar Albino Rats actuated with diabetes by administration of streptozotocin (Zanosar) was investigated. Hypoglycemic properties were evaluated after treatment of groups of male and female rats weighing $240-300 \mathrm{~g}$ with $5 \%$ and $10 \%$ extract of the herbs for 16 days resulted in significant $(p<0.05)$ reduction as measured by Glycated haemoglobin $(H b A l c)$ and Fasting blood glucose $(F B G)$ concentration. Other parameters investigated were insulin, glutathione (GSH) and Creatinine. The diabetic control rats and diabetic test rats results showed statistically significant $(p<0.05)$ difference with the control (normal) rats. These differences however vary among the herbs. T.bengwensis showed greater hypoglycemic effects than O.gratissimum. The combined treatment with the herbal mixture elucidated a synergistic effect bringing the glucose level from $18.3 \pm 0.41 \mathrm{mmol} / \mathrm{l}$ to $3.7 \pm 0.15 \mathrm{mmol} / \mathrm{l}$. Further analysis of data with a scatter plot of individual groups according to their FBG and HbAlc shows a positive linear correlation and the linear regression had a coefficient of $r=0.69$ significant at 0.01 level $(r=0.69, p<0.01$. Histological examination of the liver and pancrease were studied. Reduction in GSH concentration give credence to a possible protection for the red blood cells.
\end{abstract}

Keywords: albino rats, diabetic, herbs, hypoglycemia, induction, streptozotocin,

\section{Introduction}

The abundance of medicinal herbs in our environment and the realization that they possess active ingredients with therapeutic values has made the need for their study imperative. A scientific scrutiny of the ethnobotanical information, chemical constituent and the therapeutic application of the plant portend great opportunities for humanity. Plants play role as vessels with chemical constituents that possess pharmacological potentials and their use in management of disease has been a common practice in developing countries throughout the world. Research into medicinal plants will assist in ascertaining the efficiency of the flora as a remedy, and extend our frontiers of knowledge, their active principle, dosage and administration.

Diabetes mellitus is a heterogenous metabolic disorder characterized by chronic hyperglycemia due to dynamic interactions between varying defects of insulin secretion and action. The role of glucose in the pathologenesis of diabetic complication has attracted scientific scrutiny raising questions as to whether glucose is a reactant or an inert molecule in the pathogenesis of diabetic complications As identified by ${ }^{[1]}$ the burden of this non communicable disease on global morbidity and mortality has gained tremendous recognition. Diabetes is now known to be a leading cause of blindness, kidney failure, stroke, gangrene and loss of libido with increasing prevalence. Arising from this, the need for the survival of diabetic patient through assessment of medication, monitoring and education has become imperative.

It has been known that glycation (non-enzymatic glycosylation) is the result of a sugar molecule such as fructose or glucose binding to a protein or lipid molecule without the controlling action of an enzyme. In diabetic patients, glycation occurs excessively. Among various metabolic development implicated in the pathogenesis of diabetes vascular complication, advanced glycated en-products (AGEs) has been known to be compatible with the level of hyperglycemia. Protein molecules can irreversibly bind non-enzymatically with glucose or other sugars to form initially unstable aldimine and keto versions of the original protein and then later more stable structures. The rate at which this happens is proportional to the concentration of sugar in the surrounding medium in which the protein is found and the duration of exposure of the protein in this medium. The most important of these reactions is that with glucose and is called glycation (nonezymatic glycosylation). This is the only sugar adduction process to occur both intra and extracellularly. Diabetic patients with raised plasma glucose levels have proportionally more glcation occurring. Several researchers have carried out work on this subject. The works of ${ }^{[2,3,4,5]}$ have elucidated the need to treat these complications as an integral part of the clinical stratification of diabetic patients. Several prospective studies have been carried out which showed that intensive blood glucose control effectively reduces microvascular complication among diabetics patients. Earlier study by ${ }^{[6]}$ has elucidated a growing body of evidence to conclude tight blood glucose control is possible. As shown by ${ }^{[7,8,9,10]}$ there is growing evidence to support that inhibition of advanced glycated end product 
(AGEs) formation or blockade of their downstream signaling pathway may be a promising strategy for treatment of patients with diabetic vascular complications.

Trials are on going on substances that may be able to prevent these processes and possibly even reverse them and the prospects are promising. Some authors have earlier reported the antidiabetic and hypoglycemic properties of African Mistletoe. As shown by ${ }^{[11,12]}$, mistletoe is efficacious in the management of diabetes. This has been supported by ${ }^{[13,14]}$, who had earlier reported a reduction in some clinical parameters associated with diabetes. Previous reviews have elucidated understanding as shown by ${ }^{[15,16]}$ to the effect that Ocimum gratissimum has a dose and time dependent effect in hyperglycemic levels of streptozotocin induced rats. This research effort is on Tapinanthus bengwensis and Ocimum gratissimum aimed at determining their hyperglycemic properties on Wistar Albino Rats induced with streptozotocin.

2.1Materials

\section{Materials And Methods}

Wistar strains of Albino rats weighing between 240-300g obtained from the animal house of the Department of Zoology in the University of Port Harcourt, Rivers State, Nigeria were used for the work. A total of 102 rats were used as follows:

(a) 20 rats were used to determine the number of days in which streptozoticn-induced diabetes rats is stable.

(b) 10 rats were used to determine the hypoglycemic properties of Tapinanthus bengwensis and Ocimum gratissimum aqueous extract on streptozotocin-induced diabetic rats.

(c) 72 rats were used to determine the hypoglycemic properties of Tapinanthus bengwensis and Occimum gratissimum aqueous extract on streptotozotocin-induced diabetic rats.

\subsection{Chemicals}

(a) Streptozotocin (ZANOSAR) STZ, was obtained from Sigma chemical company, St. Lous M.O. USA. It was buffered.

(b) Daonil (Gilbenclamide phosphate) was a product of Hoescht, Ikeja, Lagos, Nigeria.

\subsection{Sources Of Plant Material}

a. i. Name: Tapinanthus bengwensis (Engl\& K Kraus) Danger

ii. Family: Loranthaceae

iii. Common Name: Mistletoe

iv. Habitat: A parasite plant

v. Locality: Citrus sinensis, Delta Park, University of Port Harcourt

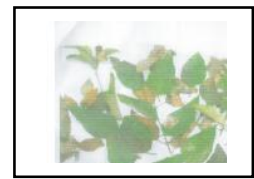

b. i. Name: Occimum gratissimum (linn)

ii. Family: Lamiaceae

iii. Common Name: Fever plant, scent leaf, tea bush, wild basil

iv. Local Name: Nchu-anwu (Igbo), Efini (Yoruba), DaidoyaTagida (Hausa), Froukena (Izon), Yanana (Epie)

\subsection{Experimental Animals}

Wistar albino rats aged (15-20 weeks) derived from a colony maintained at the animal house, at the Department of Biochemistry, Choba Park, University of Port Harcourt, Rivers State, Nigeria were used for the whole experiment. The rats weighing between $240-300 \mathrm{~g}$ were housed in cages within a temperature of $\left(25 \pm 2^{\circ} \mathrm{C}\right)$ and were divided into 8 groups of 8 animals each, and fed with standard laboratory chow (Pfizer Feeds Plc, Nigeria) and water ad libitum.

\subsection{Experimental Procedure}

Induction of diabetes mellitus was achieved through the intraperitoneal injection of streptozotocin $70 \mathrm{mg} / \mathrm{kg}$ body weight dissolved in $1.0 \mathrm{ml} / 1$ citrate buffer $\mathrm{pH} 4.5$ for two days. The animals were considered when the blood glucose values exceeded $10.0 \mathrm{mmol} / \mathrm{l}$ (range $10.0 \mathrm{mmol} / \mathrm{l}-24.3 \mathrm{mmol} / \mathrm{l}$ ), 2 weeks after STZ induction. Further confirmation by testing for glucosuria and ketonuria were also done. 


\subsection{Assay Methods}

\subsubsection{Fasting Blood Glucose}

Glucose concentration was determined after enzymatic oxidation. A violet red quinoneimine dye as an indicator was measured spectrophotometrically using UNISPEC $22 \mathrm{D}^{+}$at 520nm using Randox Kits (UK).

\subsubsection{Glycated Haemoglobing (Hba1c)}

HbA1c was determine by the enzyme endoproteinase Glu-c. In a second step the glycated and nonglycated N-terminal hexapeptides of the $\beta$-chain were separated and quantified by ion-exchange high performance liquid chromatography (HPLC-Esi/ms) approach with UV-detection. The percentage of HbA1c was determined as a ratio of the glycated to the non-glycated $\beta-\mathrm{N}$ terminal hexapeptide of haemoglobins.

\subsubsection{Insulin}

CX9 Automated machine (Beckman) was used with Beckman Assay reagent for the automated analysis of insulin. Radioactive iodine ${ }^{125}$ I-labelled insulin antibodies. Separation of bound from free ligand was accomplished by double antibody precipitation.

\subsubsection{Gsh}

The assay include monitoring and evaluation of conjugation ofdichloronitrobenzene(CDNB) with GSH at various concentration of drug extract at $340 \mathrm{~nm}$ and the optical density after every minute. The conjugate forms at $340 \mathrm{~nm}$ in the visible region.

\subsubsection{Creatinine}

Spectrophotometric method with deproteination was used in the determination of creatinine. Principle of test depend on the fact that creatinine in alkaline solution reacts with picrate to form coloured complex. The complex was read 540nm.

\section{Result}

A total of five parameters were evaluated to assess the hypoglycemic properties of the extracts along with histological studies. These were Fasting Blood Glucose (FBG), Glycated Haemoglobin (HbA1c), Insulin, Gluthanione (GSH) and Creatinine. "Tables 1-5" show the results. Whereas values for the control rats were within reference range marked variations were observed in the other groups. Test confirmed the fact that higher mean values of FBG level in diabetic (positive) control rats were resulting effects of the diabetic properties of streptozotocin induction. While the rise in FBG for the diabetic control rats were sustained, that for the control were stable while post treatment values varied. Similar observations were made for the glycated haemoglobin "Table 2". Post treatment effects of the herbs for the other parameters are shown on the tables.

Statistical evaluation of data collected were summarized as mean \pm SEM. Differences between individual groups were assessed by one way and 2-way analysis of variance (ANOVA) with significant level set at $p<0.05$. Pearson correlation coefficient was used to determine the efficacious measure of the concurrent validity of the extract.

Histological results of the pancreas and liver are shown elucidating pre and post treatment effects. From "Figure 1", the correlation of FBG and HbA1c at 0.9588 is very close to perfect. Similar comparison of the other parameters with control shows evidence of the efficacy of the extracts.

Table 1. FBG (mmol/l) Assay values for different groups

\begin{tabular}{|c|c|c|c|c|c|c|c|c|}
\hline Day & Control & $\begin{array}{c}\text { Diabetic } \\
\text { control } \\
\text { Rats } \\
\end{array}$ & $\begin{array}{l}\text { DTR on } 10 \% \\
\text { beng. }\end{array}$ & $\begin{array}{c}\text { DTR on } 10 \% \\
\text { O.grat }\end{array}$ & $\begin{array}{c}\text { DTR on } 5 \% \\
\text { T.beng }\end{array}$ & $\begin{array}{c}\text { DTR on } 5 \% \\
\text { O.grat }\end{array}$ & $\begin{array}{c}\text { DTR on } \\
\text { Daonil }\end{array}$ & $\begin{array}{c}\text { DTR on } 10 \% \\
\text { T.beng, } 10 \% \\
\text { O.grat }\end{array}$ \\
\hline 0 & $4.6 \pm 0.03^{\mathrm{a}}$ & $10.3 \pm 0.03^{b}$ & $18.3 \pm 0.06^{\mathrm{c}}$ & $16.50 \pm 0.0^{\mathrm{d}}$ & $14.40 \pm 0.05$ & $14.4 \pm 0.06$ & $18.3 \pm 0.06^{\mathrm{v}}$ & $18.3 \pm 0.41$ \\
\hline 2 & $4.7 \pm 0.03^{\mathrm{d}}$ & $10.7 \pm 0.03^{\mathrm{e}}$ & $16.3 \pm 0.03^{\mathrm{f}}$ & $16.4 \pm 0.03$ & $14.0 \pm 0.00$ & $14.3 \pm 0.03$ & $16.1 \pm 0.11$ & $17.0 \pm 0.32^{\mathrm{h}}$ \\
\hline 4 & $4.4 \pm 0.03^{\mathrm{a}}$ & $11.4 \pm 0.00^{\mathrm{c}}$ & $14.2 \pm 0.05^{\mathrm{d}}$ & $15.3 \pm 0.03$ & $13.8 \pm 0.03$ & $13.2 \pm 0.00$ & $14.2 \pm 0.11^{\mathrm{h}}$ & $15.6 \pm 0.33$ \\
\hline 6 & $4.5 \pm 0.03^{\mathrm{a}}$ & $13.3 \pm 0.08^{f}$ & $12.1 \pm 0.04$ & $15.0 \pm 0.03^{\mathrm{g}}$ & $13.4 \pm 0.03$ & $14.2 \pm 0.00^{j}$ & $12.0 \pm 0.15^{\mathrm{i}}$ & $14.0 \pm 0.51^{\mathrm{k}}$ \\
\hline 8 & $4.7 \pm 0.03^{\mathrm{m}}$ & $15.5 \pm 0.05^{\mathrm{n}}$ & $8.9 \pm 0.11$ & $15.0 \pm 0.03^{\circ}$ & $13.0 \pm 0.05$ & $14.0 \pm 0.00$ & $10.0 \pm 0.22^{\mathrm{q}}$ & $10.7 \pm 0.41$ \\
\hline 10 & $5.1 \pm 0.08^{\mathrm{s}}$ & $16.4 \pm 0.11^{\mathrm{t}}$ & $8.0 \pm 0.08^{\mathrm{u}}$ & $14.8 \pm 0.08^{\mathrm{v}}$ & $11.1 \pm 0.05^{\mathrm{w}}$ & $13.6 \pm 0.05^{\mathrm{x}}$ & $9.3 \pm 0.24$ & $8.2 \pm 0.25$ \\
\hline 12 & $5.3 \pm 0.08^{\mathrm{u}}$ & $18.2 \pm 0.08^{\mathrm{v}}$ & $8.5 \pm 0.28$ & $14.4 \pm 0.03^{\mathrm{w}}$ & $10.4 \pm 0.051$ & $13.3 \pm 0.03^{x}$ & $7.0 \pm 0.27^{\mathrm{t}}$ & $5.7 \pm 0.13$ \\
\hline 14 & $4.6 \pm 0.12^{\mathrm{a}}$ & $22.3 \pm 0.08^{b}$ & $6.3 \pm 0.00^{\mathrm{e}}$ & $14.0 \pm 0.00^{\mathrm{f}}$ & $10.0 \pm 0.06^{\mathrm{g}}$ & $13.1 \pm 0.13^{\mathrm{a}}$ & $5.2 \pm 0.11$ & $4.4 \pm 0.14$ \\
\hline 16 & $4.7 \pm 0.08^{\mathrm{a}}$ & $24.3 \pm 0.93^{\mathrm{d}}$ & $5.6 \pm 0.03$ & $13.3 \pm 0.03^{\mathrm{g}}$ & $7.6 \pm 0.05$ & $12.7 \pm 0.12$ & $4.4 \pm 0.27$ & $3.7 \pm 0.15$ \\
\hline
\end{tabular}

Values are mean \pm SEM of triplicate determination. Values on the same row having the same superscript are not significantly different from each other. DTR - Diabetic Test Rats, T.beng - Tapinanthus bengwensis, O.grat Ocimum gratissimum 
Table 2. HbA1c (\%) Assay values for different groups

\begin{tabular}{|c|c|c|c|c|c|c|c|c|}
\hline $\begin{array}{c}\text { Da } \\
\text { y }\end{array}$ & Control & $\begin{array}{c}\text { Diabetic } \\
\text { control } \\
\text { rats }\end{array}$ & $\begin{array}{c}\text { DTR on 10\% } \\
\text { beng. }\end{array}$ & $\begin{array}{c}\text { DTR on 10\% } \\
\text { O.grat }\end{array}$ & $\begin{array}{c}\text { DTR on 5\% } \\
\text { T.beng }\end{array}$ & $\begin{array}{c}\text { DTR on 5\% } \\
\text { O.grat }\end{array}$ & $\begin{array}{c}\text { DTR on } \\
\text { Daonil }\end{array}$ & $\begin{array}{c}\text { DTR on 10\% } \\
\text { T.beng, 10\% } \\
\text { O.grat }\end{array}$ \\
\hline 0 & $4.3 \pm 0.03^{\mathrm{m}}$ & $10.4 \pm 0.08^{\mathrm{n}}$ & $11.6 \pm 0.03$ & $12.5 \pm 0.85^{\mathrm{o}}$ & $12.0 \pm 0.02$ & $11.6 \pm 0.02$ & $11.8 \pm 0.71^{\mathrm{v}}$ & $13.3 \pm 0.51^{\mathrm{h}}$ \\
\hline 2 & $4.4 \pm 0.06^{\mathrm{a}}$ & $10.0 \pm 0.57^{\mathrm{b}}$ & $11.0 \pm 0.75$ & $12.4 \pm 0.02^{\mathrm{o}}$ & $11.6 \pm 0.04$ & $11.5 \pm 0.82$ & $11.5 \pm 0.82^{\mathrm{h}}$ & $12.0 \pm 0.31$ \\
\hline 4 & $4.5 \pm 0.05^{\mathrm{s}}$ & $12.2 \pm 0.10^{\mathrm{t}}$ & $10.1 \pm 0.05^{\mathrm{u}}$ & $11.6 \pm 0.04$ & $11.3 \pm 0.05$ & $11.5 \pm 0.03$ & $11.0 \pm 0.7^{\mathrm{w}}$ & $11.0 \pm 0.8$ \\
\hline 6 & $4.3 \pm 0.03^{\mathrm{m}}$ & $14.3 \pm 0.05^{\mathrm{h}}$ & $9.2 \pm 0.08^{\mathrm{r}}$ & $11.5 \pm 0.04 \mathrm{~s}$ & $11.0 \pm 0.02$ & $11.3 \pm 0.83$ & $10.1 \pm 0.8^{\mathrm{w}}$ & $9.3 \pm 0.21$ \\
\hline 8 & $4.4 \pm 0.40^{\mathrm{x}}$ & $15.6 \pm 0.03^{\mathrm{y}}$ & $9.7 \pm 0.36^{\mathrm{z}}$ & $11.0 \pm 0.03^{\mathrm{o}}$ & $10.8 \pm 0.04$ & $11.0 \pm 0.06$ & $10.1 \pm 0.82^{\mathrm{q}}$ & $8.0 \pm 0.41^{\mathrm{v}}$ \\
\hline 10 & $4.6 \pm 0.03^{\mathrm{a}}$ & $15.9 \pm 0.35^{\mathrm{b}}$ & $6.8 \pm 0.05^{\mathrm{c}}$ & $10.5 \pm 0.05^{\mathrm{d}}$ & $10.5 \pm 0.6$ & $10.8 \pm 0.85$ & $5.0 \pm 0.88$ & $6.2 \pm 0.11$ \\
\hline 12 & $4.4 \pm 0.40^{\mathrm{e}}$ & $16.3 \pm 0.03^{\mathrm{t}}$ & $6.0 \pm 0.03^{\mathrm{g}}$ & $9.4 \pm 0.06$ & $9.4 \pm 0.03$ & $10.5 \pm 0.06^{\mathrm{h}}$ & $4.2 \pm 0.86$ & $5.1 \pm 0.23$ \\
\hline 14 & $4.4 \pm 0.30^{\mathrm{h}}$ & $17.4 \pm 0.02^{\mathrm{k}}$ & $5.5 \pm 0.03$ & $9.0 \pm 0.04$ & $8.0 \pm 0.03$ & $10.0 \pm 0.06^{\mathrm{i}}$ & $3.2 \pm 0.42$ & $4.6 \pm 0.22$ \\
\hline 16 & $4.4 \pm 0.57$ & $18.3 \pm 0.04^{\mathrm{e}}$ & $4.0 \pm 0.03^{\mathrm{b}}$ & $8.9 \pm 0.03^{\mathrm{b}}$ & $7.2 \pm 0.02$ & $9.7 \pm 0.04^{\mathrm{c}}$ & $3.2 \pm 0.42$ & $3.0 \pm 0.23$ \\
\hline
\end{tabular}

Values are mean + SEM of triplicate determination. Values on the same row having the same superscript are not significantly different from each other. DTR - Diabetic Test Rats, T.beng - Tapinanthus bengwensis, O.grat Ocimum gratissimum

Table 3. Insulin (uU/mol) Assay values for different groups

\begin{tabular}{|c|c|c|c|c|c|c|c|c|}
\hline Day & Control & $\begin{array}{c}\text { Diabetic } \\
\text { control } \\
\text { Rats }\end{array}$ & $\begin{array}{c}\text { DTR on 10\% } \\
\text { beng. }\end{array}$ & $\begin{array}{c}\text { DTR on 10\% } \\
\text { O.grat }\end{array}$ & $\begin{array}{c}\text { DTR on 5\% } \\
\text { T.beng }\end{array}$ & $\begin{array}{c}\text { DTR on 5\% } \\
\text { O.grat }\end{array}$ & $\begin{array}{c}\text { DTR on } \\
\text { Daonil }\end{array}$ & $\begin{array}{c}\text { DTR on 10\% } \\
\text { T.beng, 10\% } \\
\text { O.grat }\end{array}$ \\
\hline 0 & $5.3 \pm 0.43^{\mathrm{a}}$ & $5.8 \pm 0.22^{\mathrm{a}}$ & $0.4 \pm 0.18^{\mathrm{b}}$ & $0.63 \pm 0.13$ & $0.5 \pm 0.16$ & $0.6 \pm 012$ & $0.5 \pm 0.17$ & $0.5 \pm 0.12^{\mathrm{b}}$ \\
\hline 2 & $5.4 \pm 0.22^{\mathrm{b}}$ & $4.2 \pm 0.11$ & $0.6 \pm 0.11^{\mathrm{c}}$ & $0.60 \pm 0.15$ & $0.5 \pm 0.17$ & $0.6 \pm 0.12^{\mathrm{f}}$ & $0.8 \pm 0.18$ & $0.5 \pm 0.13^{\mathrm{e}}$ \\
\hline 4 & $4.8 \pm 0.42^{\mathrm{a}}$ & $4.0 \pm 0.12$ & $0.6 \pm 0.11^{\mathrm{c}}$ & $0.7 \pm 0.16$ & $0.6 \pm 0.14$ & $0.7 \pm 0.11$ & $0.9 \pm 0.21$ & $0.7 \pm 0.12^{\mathrm{s}}$ \\
\hline 6 & $3.2 \pm 0.42^{\mathrm{a}}$ & $3.0 \pm 0.13$ & $1.3 \pm 0.13^{\mathrm{d}}$ & $0.8 \pm 0.27^{\mathrm{e}}$ & $0.7 \pm 0.15$ & $0.8 \pm 0.13$ & $1.2 \pm 0.23_{\mathrm{r}}$ & $1.3 \pm 0.31^{\mathrm{d}}$ \\
\hline 8 & $4.8 \pm 0.58^{\mathrm{f}}$ & $2.6 \pm 0.11^{\mathrm{g}}$ & $1.5 \pm 0.18^{\mathrm{a}}$ & $0.93 \pm 0.12$ & $0.9 \pm 0.17$ & $0.8 \pm 0.13$ & $1.6 \pm 0.22^{\mathrm{k}}$ & $1.7 \pm 0.12^{\mathrm{k}}$ \\
\hline 10 & $7.3 \pm 0.22^{\mathrm{w}}$ & $1.4 \pm 0.12^{\mathrm{f}}$ & $2.0 \pm 0.22$ & $1.1 \pm 0.11$ & $0.9 \pm 0.17^{\mathrm{y}}$ & $0.8 \pm 0.13$ & $2.3 \pm 0.24^{\mathrm{r}}$ & $1.9 \pm 0.32$ \\
\hline 12 & $6.5 \pm 0.22^{\mathrm{w}}$ & $1.0 \pm 0.11^{\mathrm{q}}$ & $2.3 \pm 0.11^{\mathrm{r}}$ & $1.0 \pm 0.12^{\mathrm{f}}$ & $1.0 \pm 0.12^{\mathrm{f}}$ & $0.9 \pm 0.11$ & $3.2 \pm 0.18$ & $2.4 \pm 0.11$ \\
\hline 14 & $6.8 \pm 0.27^{\mathrm{v}}$ & $0.5 \pm 0.26^{\mathrm{r}}$ & $3.0 \pm 0.12^{\mathrm{f}}$ & $1.2 \pm 0.13^{\mathrm{p}}$ & $1.2 \pm 0.15$ & $0.9 \pm 0.11$ & $3.5 \pm 0.16$ & $2.6 \pm 0.14^{\mathrm{x}}$ \\
\hline 16 & $6.7 \pm 0.02^{\mathrm{f}}$ & $0.2 \pm 0.17^{\mathrm{d}}$ & $3.3 \pm 0.25^{\mathrm{r}}$ & $1.3 \pm 0.11^{\mathrm{r}}$ & $1.3 \pm 0.16$ & $1.1 \pm 0.12$ & $3.8 \pm 0.13$ & $3.6 \pm 9.16$ \\
\hline
\end{tabular}

Values are mean \pm SEM of triplicate determination. Values on the same row having the same superscript are not significantly different from each other. DTR - Diabetic Test Rats, T.beng - Tapinanthus bengwensis, O.grat Ocimum gratissimum

Table 4.Glutathione (GSH) (uu/molHb) Assay values for different groups

\begin{tabular}{|c|c|c|c|c|c|c|c|c|}
\hline Day & Control & $\begin{array}{c}\text { Diabetic } \\
\text { control } \\
\text { Rats }\end{array}$ & $\begin{array}{c}\text { DTR on 10\% } \\
\text { beng. }\end{array}$ & $\begin{array}{c}\text { DTR on 10\% } \\
\text { O.grat }\end{array}$ & $\begin{array}{c}\text { DTR on 5\% } \\
\text { T.beng }\end{array}$ & $\begin{array}{c}\text { DTR on 5\% } \\
\text { O.grat }\end{array}$ & $\begin{array}{c}\text { DTR on } \\
\text { Daonil }\end{array}$ & $\begin{array}{c}\text { DTR on 10\% } \\
\text { T.beng, 10\% } \\
\text { O.grat }\end{array}$ \\
\hline 0 & $0.40+0.0^{\mathrm{a}}$ & $0.32 \pm 0.11^{\mathrm{b}}$ & $0.33 \pm 0.00$ & $0.53 \pm 0.01^{\mathrm{c}}$ & $0.35 \pm 0.02$ & $0.36 \pm 0.11$ & $0.34+0.11$ & $0.36 \pm 0.02$ \\
\hline 2 & $0.44+0.0^{\mathrm{a}}$ & $0.30 \pm 0.12$ & $0.44 \pm 0.12^{\mathrm{a}}$ & $0.3 \pm 0.02^{\mathrm{b}}$ & $0.30 \pm 0.02^{\mathrm{b}}$ & $0.35 \pm 0.12$ & $0.34+0.12$ & $0.4 \pm 0.02$ \\
\hline 4 & $0.42+0.01$ & $0.33 \pm 0.01$ & $0.48 \pm 0.02$ & $0.37 \pm 0.03$ & $0.30 \pm 0.00$ & $0.33 \pm 0.00$ & $0.4+0.01$ & $0.47 \pm 0.03$ \\
\hline 6 & $0.36+0.0^{\mathrm{a}}$ & $0.30 \pm 0.01$ & $0.52 \pm 0.02^{\mathrm{b}}$ & $0.39 \pm 0.02$ & $0.28 \pm 0.00^{\mathrm{c}}$ & $0.31 \pm 0.01$ & $0.5+0.11 \mathrm{~d}$ & $0.5 \pm 0.04^{\mathrm{d}}$ \\
\hline 8 & $0.37+0.0^{\mathrm{e}}$ & $0.25 \pm 0.12^{\mathrm{f}}$ & $0.55 \pm 0.22^{\mathrm{g}}$ & $0.32 \pm 0.02$ & $0.34 \pm 0.03$ & $0.33 \pm 0.02$ & $0.68+0.171$ & $0.8 \pm 0.04^{\mathrm{i}}$ \\
\hline 10 & $0.41+0.0^{\mathrm{w}}$ & $0.20 \pm 0.13^{\mathrm{l}}$ & $0.65 \pm 0.02^{\mathrm{q}}$ & $0.38 \pm 0.02^{\mathrm{r}}$ & $0.54 \pm 0.02^{\mathrm{t}}$ & $0.31 \pm 0.11^{\mathrm{u}}$ & $0.93+0.21$ & $0.9 \pm 0.12^{\mathrm{w}}$ \\
\hline 12 & $0.42+0.0^{\mathrm{r}}$ & $0.15 \pm 0.13^{\mathrm{s}}$ & $0.77 \pm 0.02^{\mathrm{t}}$ & $0.38 \pm 0.02^{\mathrm{g}}$ & $0.52 \pm 0.02^{\mathrm{l}}$ & $0.33 \pm 0.01^{\mathrm{i}}$ & $0.93+0.12$ & $1.0 \pm 0.04^{\mathrm{k}}$ \\
\hline 14 & $0.43+0.00^{\mathrm{l}}$ & $0.09 \pm 0.12^{\mathrm{m}}$ & $0.82 \pm 0.12^{\mathrm{n}}$ & $0.39 \pm 0.02^{\mathrm{o}}$ & $0.52 \pm 0.02^{\mathrm{p}}$ & $0.32 \pm 0.12^{\mathrm{q}}$ & $1.3+0.11 \mathrm{~s}$ & $1.1 \pm 0.11$ \\
\hline 16 & $0.44+0.0^{\mathrm{k}}$ & $0.10 \pm 0.11^{1}$ & $0.91 \pm 0.02^{\mathrm{m}}$ & $0.39 \pm 0.02^{\mathrm{n}}$ & $0.50 \pm 0.02^{\mathrm{p}}$ & $0.30 \pm 0.14^{\mathrm{r}}$ & $1.5+0.31$ & $1.3 \pm 0.12^{\mathrm{v}}$ \\
\hline
\end{tabular}

Values are mean \pm SEM of triplicate determination. Values on the same row having the same superscript are not significantly different from each other. DTR - Diabetic Test Rats, T.beng - Tapinanthus bengwensis, O.grat Ocimum gratissimum

Table 5.Creatinine ( $\mu \mathrm{mol} / \mathrm{l})$ Assay values for different groups

\begin{tabular}{|c|c|c|c|c|c|c|c|c|}
\hline Day & Control & $\begin{array}{c}\text { Diabetic } \\
\text { control } \\
\text { Rats }\end{array}$ & $\begin{array}{c}\text { DTR on 10\% } \\
\text { beng. }\end{array}$ & $\begin{array}{c}\text { DTR on 10\% } \\
\text { O.grat }\end{array}$ & $\begin{array}{c}\text { DTR on 5\% } \\
\text { T.beng }\end{array}$ & $\begin{array}{c}\text { DTR on 5\% } \\
\text { O.grat }\end{array}$ & $\begin{array}{c}\text { DTR on } \\
\text { Daonil }\end{array}$ & $\begin{array}{c}\text { DTR on } \\
10 \% \\
\text { T.beng, } \\
10 \% \text { O.grat }\end{array}$ \\
\hline 0 & $85 \pm 4.4^{\mathrm{a}}$ & $135 \pm 0.85^{\mathrm{b}}$ & $140 \pm 2.3$ & $135 \pm 4.2$ & $140 \pm 3.3^{\mathrm{c}}$ & $140 \pm 4.2$ & $140 \pm 5.3^{\mathrm{c}}$ & $140 \pm 5.0$ \\
\hline 2 & $90 \pm 5.8^{\mathrm{m}}$ & $140 \pm 4.0^{\mathrm{n}}$ & $76 \pm 0.12^{\mathrm{o}}$ & $130 \pm 4.2^{\mathrm{p}}$ & $145 \pm 4.6^{\mathrm{q}}$ & $145 \pm 4.4$ & $135 \pm 2.5$ & $143 \pm 4.8$ \\
\hline 4 & $95 \pm 3.4^{\mathrm{r}}$ & $139 \pm 2.5^{\mathrm{s}}$ & $145 \pm 2.5^{\mathrm{t}}$ & $135 \pm 3.6$ & $145 \pm 4.2^{\mathrm{t}}$ & $145 \pm 4.2$ & $130 \pm 2.3^{\mathrm{v}}$ & $145 \pm 5.0^{\mathrm{t}}$ \\
\hline 6 & $85.0 \pm 0.87^{\mathrm{s}}$ & $140 \pm 2.0^{\mathrm{n}}$ & $135 \pm 2.2^{\mathrm{w}}$ & $135 \pm 3.8$ & $150 \pm 4.5^{\mathrm{x}}$ & $150 \pm 4.5^{\mathrm{x}}$ & $130 \pm 2.5^{\mathrm{y}}$ & $148 \pm 4.0^{\mathrm{z}}$ \\
\hline 8 & $100 \pm 0.85^{\mathrm{a}}$ & $150 \pm 2.0^{\mathrm{b}}$ & $130 \pm 2.3^{\mathrm{d}}$ & $140 \pm 3.8^{\mathrm{f}}$ & $155 \pm 3.2^{\mathrm{g}}$ & $153 \pm 3.2$ & $120 \pm 2.5$ & $130 \pm 3.8$ \\
\hline 10 & $95 \pm 0.59^{\mathrm{x}}$ & $145 \pm 2.4^{\mathrm{y}}$ & $130 \pm 2.3^{\mathrm{x}}$ & $130 \pm 3.3$ & $150 \pm 3.3^{\mathrm{m}}$ & $150 \pm 3.3$ & $100 \pm 0.5^{\mathrm{o}}$ & $140 \pm 2.5^{\mathrm{p}}$ \\
\hline 12 & $95 \pm 0.85^{\mathrm{l}}$ & $155 \pm 2.2^{\mathrm{j}}$ & $120 \pm 2.0^{\mathrm{k}}$ & $135 \pm 2.5^{\mathrm{l}}$ & $140 \pm 4.2^{\mathrm{m}}$ & $140 \pm 4.2$ & $80 \pm 2.4$ & $130 \pm 3.0^{\mathrm{p}}$ \\
\hline 14 & $85 \pm 0.80^{\mathrm{q}}$ & $150 \pm 3.3^{\mathrm{r}}$ & $100 \pm 2.5^{\mathrm{s}}$ & $130 \pm 3.4^{\mathrm{t}}$ & $145 \pm 5.2^{\mathrm{u}}$ & $145 \pm 5.2^{\mathrm{u}}$ & $75 \pm 2.3$ & $120 \pm 2.2^{\mathrm{w}}$ \\
\hline 16 & $85 \pm 2.5^{\mathrm{s}}$ & $165 \pm 2.5^{\mathrm{t}}$ & $110 \pm 3.5^{\mathrm{u}}$ & $125 \pm 3.3^{\mathrm{v}}$ & $135 \pm 5.3^{\mathrm{w}}$ & $135 \pm 5.3$ & $60 \pm 3.4^{\mathrm{x}}$ & $100 \pm 2.1^{\mathrm{y}}$ \\
\hline
\end{tabular}

Values are mean \pm SEM of triplicate determination. Values on the same row having the same superscript are not significantly different from each other. DTR - Diabetic Test Rats, T.beng - Tapinanthus bengwensis, O.grat Ocimum gratissimum 

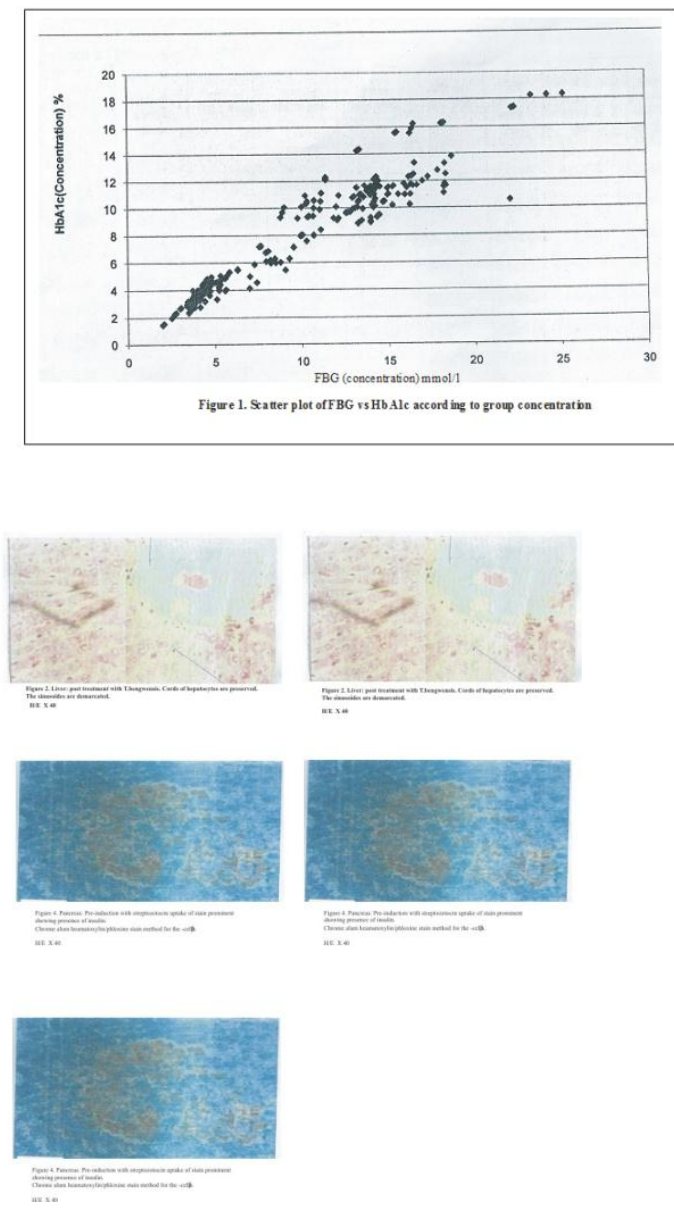

\section{Discussion}

Several local herbs are being used by the population as alternative therapy for the treatment of diabetes. Most of the herbs have not been subjected to scientific scrutiny to determine their potency. This study has brought to the fore the need to thoroughly examine these herbs for their efficacy. Swantston-Flatt et al (1989) had earlier reported a reduction in some clinical parameters associated with diabetes after chronic administration of mistletoe $(6.25 \%$ by weight of diet, $1 \mathrm{~g} / 400 \mathrm{ml}$ infusion in place of drinking water) ameliorated symptoms of polydipsia, hyperphagia and body weight loss in severely hyperglycemic streptotozotin-diabetic mice. However significant decrease of plasma glucose was not demonstrated in this insulin deficient model. In our study, a marked decrease in plasma glucose has been demonstrated. It is possible to explain that species differences in the plant used may have accounted for the variation of results. This work has given credence to earlier study of ${ }^{[11,12]}$ and ${ }^{[13]}$ in which the antidiabetic and hypoglycemic properties were reported.

Phytochemical analysis of mistletoe as earlier reported by previous workers ${ }^{[11,18]}$ have attributed the hypoglycemic properties to the presence of some constituents notably saponins, tannins, lectins and cholin derivatives. It was suggested that tannins could account partly for the observed reduction of glucose by the same mechanism that makes them antinutrients. The observed hypoglycemic properties must have been potentiated by the behaviours of the several secondary plants metabolites. It is known that cardiac glycosides, resins, reducing sugars, steroids, coumarines, terpenoides, flavonoids and other plant metabolites including arginine and glutamic acid possesses hypoglycemic effect in various experimental animals. The effect of flavonoids quercetin and ferulic acid on pancreatic $\beta$-cells leading to their proliferation and secretion of more insulin have been proposed. ${ }^{[19,20,21]}$ and ${ }^{[22]}$ have suggested $\beta$-cell recovery as the mechanism by which hyperglemia caused by streptotozotocin reduces glucose. As shown by ${ }^{[23]}$ aqueous extract of mistletoe enhanced insulin secretion and mimicked the effect of insulin on glucose metabolism. Such dual pancreatice and extrapancreatice action would prove to be an important advance on existing therapies used to treat and control diabetes, such as hypoglycemic drugs (which act by either enhancing insulin secretion or by improving the action of insulin). These combined findings even with mistletoe and Ocimum gratissimum illustrate the enormous potential of these plants for use as possible dietary adjuncts and the discovery of natural products for diabetes therapy. 
This work has elucidated antiglycation as a potential hypoglycemic property of T.bengwensis and O.gratissimum and strongly support the work of some early authors. It has been shown by ${ }^{[7,8,10]}$ growing evidence to support that inhibition of advance glycated end-products (AGEs) or blockade of their downstream signaling pathway may be a promising strategy for treatment of patient with diabetic complications.

In the present studies, statistical analysis indicates that significant differences at $(\mathrm{p}<0.05)$ existed between tested groups of rat for various parameters see "Table 1-5". Of particular importance is the value of glycated haemoglobin (HbA1c) which correlated linearly with fasting blood glucose (FBG). Further analysis of the data with a scatter plot of individual groups according to their FBG and HbA1c shows a positive linear correlation and the linear regression had a coefficient of $r=0.69$ significant at $0.01(r=0.69, p<0.01)$ see "Table 1,2 ". The fact that a significant decrease in both the glucose and HbA1c was noticed after treatment with the extract especially with T.bengwensis has exhibited a potent hypoglycemic property of the herbs.

It is possible that the synthesis of HbA1c may represent a model reaction to explain the biochemical basis for many of the long term sequlae of diabetes. The tissues that suffer the most noticeable dysfunction in diabetes (e.g. retina, lens, peripheral nerves, kidney) appear to be insulin independent for glucose uptake. Perhaps in diabetes intra-cellular proteins of these tissues undergo excess non-enzymatic glycosylation analogous to that seen within red cells. Such glycosylation might alter the enzymatic activity, solubility, antigenicity and other function of protein and thereby result in the observed clinical dysfunction. Again HbAlc has decreased reactivity with 2,3-diphosphoglycerate so red cells of diabetics will have increased affinity for oxygen.

This study included the analysis of insulin and GSH parameters to investigate the hypoglycemic properties of the herbs. As shown in table 3 and 4 respectively for insulin and GSH, a significant $(\mathrm{p}<0.05)$ was observed for both parameters. While there was an improvement in the insulin concentration, an increase in the GSH levels was also noticed. The increased concentration of GSH shows that the redox potentials of the red blood cell and other cells could be enhanced. Reduced concentration of GSH would mean an exposure of the cells to free radicals/antioxidants and thus to oxidant damage with accompanying complications. An improvement in the creatinine concentration was noticed particularly with the T.bengwensis treatment laying credence to the fact that there is a beneficial effect on the liver and pancreas. The histology of the tissues revealed moderate recovery especially for the kidney and pancreas. The correlation coefficient done for all tested groups shows that the hypoglycemic properties exhibited by the herbs were both concentration and time dependent with T.bengwensis showing stronger potentials.

Diabetes mellitus is increasingly being recognized as a major public health problem in developing countries. Although our understanding of this endemic non-communicable disease has increased overtime, the complication remains a problem. This study has elucidated the hypoglycemic properties of our local herbs and the need for further evaluations, modification and use as a means to effective monitoring, control and management of diabetes mellitus patients.

\section{Conclusion}

This study has extended our level of understanding on the interplay of biochemical molecules in the diabetic process. The need for a more incisive study of local herbs has become imperative. Further analysis of phytochemistry of these herbs are suggested for us to have an increased understanding of the potency and efficacy of the herbs used for this study.

\section{References}

[1] Odia, O.J., Wokoma, F.S, Mortality Pattern in Medical Wards of a Nigerian Teaching Hospital. Orient. 4, 1982, 96-101.

[2] Brown Lee, M, Biochemistry and Molecular Cell Biology of Diabetic Complications. Nature 414, 2001, 813-820.

[3] Brucala, A. and Cerami, A, Advanced glycosylation. Chemistry, Biology and Implications for Diabetes and aging. Av. Pharmacol 23, 1992, 1-34.

[4] Standing, S.J., and Taylor, R.P.,Glycated in assessment of high capacity liquid chromatography and immunoassay in diabetic rats. Phytother Res. 18, 1992, 310-314.

[5] Philip, A., Floeye, A., and Jansson, U, Progression of Diabetic Nephropathy from cell studies and animal models. Kidney Blood Press Res. 22, 1999, 81-97

[6] Wincour, P.H. heart disease and diabetes. (Ed. Fisher M, London: Martin Dienitz Ltd, 2003)

[7] Buciareli, L.G.; Wendt, T. and Qu, W, Rage Blockade Stabilize established artheriosclerosis in diabetic apolipoprotien E-Null mice. Circulation. 106, 2002, 2827-35.

[8] Naka, Y., Buciareli, L.G. and Wendt, Rage Axis. Animal Model and Novel insights into vascular complications of Diabetes.ArteriosterVasc Biol. 24, 2004, 1342-1349.

[9] Melpomemi, P, Glucose, Advanced Glycated End products, and diabetic complications: What is New and What Works. Clinical Diabetes. 21(4), 2003, 148-149

[10] Vlassara, H, Advanced Glycation in Health and Disease: Role of the Modern Environment. Annals of the New York Academy of Science. 1043, 2005, 452-460 doi:101196/annals.

[11] Obatomi, D.K., Bikomo, E.Q., and Victor, J.T,Antidiabetic properties of the African Mistletoe in streptozotocin-induced diabetic rat. J. Ethnopharmacol. 81, 1994, 231-237 
[12] Didem, D.O., Mustata, A., Nilufers, F., and Erdem, Y, Evaluation of hypoglycemic effect and antioxidant activity of three viscum album subspecies (European mistletoe) in streptozotocin-diabetic rats. J. Ethnopharmacol. 98, 2005, 95-102

[13] George, G.S., Ibeh, G.O., Uwakwe, A.A. Hypoglycemic effect of Aqueous Extract of mistletoe (Tapinanthusbengwensis) on streptozotocin induced diabetic rats. Journal of Research in Bioscience, .2, 2006, ISSBN 1597-801X

[14] Swanston-Flatt, S.K., Day, C., Baily, C.J. and Flatt, P.R. Evolution of traditional plant treatment for diabetics. Studies in streptozotocin diabetic mice. In ActaDiabetol Lat. 26(1), 1989, 51-5.

[15] Agnanient, H, Arguilet, J., Bessieve, J.W., and Menut, C. Aromatic plant of tropical Central Africa. Part XL-VII. Chemical and Biological Investigation of essential oil of Occimum species from Gaboni. J. ESS. Oil. Res. Abstract, 2005.

[16] Mohammed, A., Tanko, Y., Okasha, A.M., Magazi, R.A., and Yaro., A.H. Effects of acqueous extract of Occimumgratissimum on blood glucose levels of streptozotocin induced wistar rats. African Journal of Biotechnology. 6(18), 2007, 2087-2090.

[17] Litwak, K.N., Cefalu, W.T., Wagner, J.D. Strepzotocin induced diabetes mellitus in cynomolgus monkeys: changes in carbohydrate metabolism skin glycation and pancreation islets. Lab Anim. Sci., 48, 1998, 172-778.

[18] Alessi, D., Beger, A., Cepko, C., Colombet, I., Costigan, M., and Glunezoglu, A., Mistletoe. Complementary Alternative Medicine Review. 1-4, 2003.

[19] Kako, M., Mirura, T., Nishiyama.,Ichimara, M., and Kato, A., Hypoglycemic activity of some tritenoid glycosides. J. Nat. prod. 60, 1997, 604-605.

[20] Okomoto, K., Experimental production of diabetes in diabetic mellitus. Theory and practice. EdsEllenberge M. and Rifkin H. Blackinson Publications McGraw-Hill Book Company. New York, USA; 1970.

[21] Mahesh, T., and Menon, P.V. Quercetin alleviates oxidative stress in streptozotocin induced diabetic rats. Phythe. Res. 18, 2004, 123-127.

[22] Sri-Balasubashimi, M., Rukkumani, R., Vismanathan, P., and Menon, P.V. Ferulic acid alleviates lipid peroxidation in diabetic rats. Phythother Res. 18, 2004, 310-314.

[23] Gray, A.M., and Flatt, P.R. Pancreatice and extra pancreatic effect of the traditional antidiabetic plant medica sativa (Lucerne). The British Journal of Nutrition. 78(2), 1998, 325-334. 